\title{
A Short Note on Wavelet Frames Based on FMRA on Local Fields
}

\author{
M. Younus Bhat 1 iD \\ Department of Mathematical Sciences, Islamic University of Science and Technology, Awantipora, Pulwama, \\ Jammu and Kashmir 192122, India
}

Correspondence should be addressed to M. Younus Bhat; gyounusg@gmail.com

Received 14 July 2020; Revised 2 September 2020; Accepted 11 October 2020; Published 2 November 2020

Academic Editor: Asghar Rahimi

Copyright (C) 2020 M. Younus Bhat. This is an open access article distributed under the Creative Commons Attribution License, which permits unrestricted use, distribution, and reproduction in any medium, provided the original work is properly cited.

The concept of frame multiresolution analysis (FMRA) on local fields of positive characteristic was given by Shah in his paper, Frame Multiresolution Analysis on Local Fields published by Journal of Operators. The author has studied the concept of minimum-energy wavelet frames on these prime characteristic fields. We continued the studies based on frame multiresolution analysis and minimum-energy wavelet frames on local fields of positive characteristic. In this paper, we introduce the notion of the construction of minimum-energy wavelet frames based on FMRA on local fields of positive characteristic. We provide a constructive algorithm for the existence of the minimum-energy wavelet frame on the local field of positive characteristic. An explicit construction of the frames and bases is given. In the end, we exhibit an example to illustrate our algorithm.

\section{Introduction}

Let $K$ be a field and a topological space. Then, $K$ is called a local field if both $K^{+}$and $K^{*}$ are locally compact abelian groups, where $K^{+}$and $K^{*}$ denote the additive and multiplicative groups of $K$, respectively. If $K$ is any field and is endowed with the discrete topology, then $K$ is a local field. Furthermore, if $K$ is connected, then $K$ is either $\mathbb{R}$ or $\mathbb{C}$. If $K$ is not connected, then it is totally disconnected. Hence, by a local field, we mean a field $K$ which is locally compact, nondiscrete, and totally disconnected. The $p$-adic fields are examples of local fields. For more details, refer [1]. In the rest of this paper, we use the symbols $\mathbb{N}, \mathbb{N}_{0}$ and $\mathbb{Z}$ to denote the sets of natural and nonnegative integers and integers, respectively.

Let $K$ be a local field. Let $\mathrm{d} x$ be the Haar measure on the locally compact abelian group $K^{+}$. If $\alpha \in K$ and $\alpha \neq 0$, then $\mathrm{d}(\alpha x)$ is also a Haar measure. Let $\mathrm{d}(\alpha x)=|\alpha| \mathrm{d} x$. We call $|\alpha|$ the absolute value of $\alpha$. Moreover, the map $x \longrightarrow|x|$ has the following properties: (a) $|x|=0$ if and only if $x=0$; (b) $|x y|=|x \| y|$ for all $x, y \in K$; and (c) $|x+y| \leq \max \{|x|,|y|\}$ for all $x, y \in K$. Property (c) is called the ultrametric inequality. The set $\mathfrak{D}=\{x \in K:|x| \leq 1\}$ is called the ring of integers in $K$. Define $\mathfrak{B}=\{x \in K:|x|<1\}$. The set $\mathfrak{B}$ is called the prime ideal in $K$. The prime ideal in $K$ is the unique maximal ideal in $\mathfrak{D}$, and therefore $\mathfrak{B}$ is principal ideal as well as prime ideal. Since the local field $K$ is totally disconnected, there exists an element of $\mathfrak{B}$ of maximal absolute value. Let $\mathfrak{P}$ be a fixed element of the maximum absolute value in $\mathfrak{B}$. Such an element is called a prime element of $K$. Therefore, for such ideal $\mathfrak{B}$ in $\mathfrak{D}$, we have $\mathfrak{B}=\langle\mathfrak{P}\rangle=\mathfrak{P} \mathfrak{D}$. As it was proved in [1], the set $\mathfrak{D}$ is compact and open. Hence, $\mathfrak{B}$ is compact and open. Therefore, the residue space $\mathfrak{D} / \mathfrak{B}$ is isomorphic to a finite field $\operatorname{GF}(q)$, where $q=p^{k}$ for some prime $p$ and $k \in \mathbb{N}$.

Let $\mathfrak{D}^{*}=\mathfrak{D} \backslash \mathfrak{B}=\{x \in K:|x|=1\}$. Then, it can be proved that $\mathfrak{D}^{*}$ is a group of units in $K^{*}$, and if $x \neq 0$, then we may write $x=\mathfrak{P}^{k} x l, x \in \in \mathfrak{D}^{*}$. For a proof of this fact, refer [1]. Moreover, each $\mathfrak{B}^{k}=\mathfrak{P}^{k} \mathfrak{D}=\left\{x \in K:|x|<q^{-k}\right\}$ is a compact subgroup of $K^{+}$and usually known as the fractional ideals of $K^{+}$. Let $\mathcal{U}=\left\{a_{i}\right\}_{i=0}^{q-1}$ be any fixed full set of coset representatives of $\mathfrak{B}$ in $\mathfrak{D}$; then, every element $x \in K$ can be expressed uniquely as $x=\sum_{\ell=k}^{\infty} c_{\ell} \mathfrak{P}^{\ell}$ with $c_{\ell} \in \mathcal{U}$. Let $\chi$ be a fixed character on $K^{+}$that is trivial on $\mathfrak{D}$ but is nontrivial on $\mathfrak{B}^{-1}$. Therefore, $\chi$ is a constant on cosets of $\mathfrak{D}$, so if $y \in \mathfrak{B}^{k}$, then $\chi_{y}(x)=\chi(y x), \quad x \in K$. Suppose that $\chi_{u}$ is any character on $K^{+}$; then, clearly, the restriction $\chi_{u} \mid \mathfrak{D}$ is also a character on $\mathfrak{D}$. Therefore, if $\left\{u(n): n \in \mathbb{N}_{0}\right\}$ is a complete list of the distinct coset representative of $\mathfrak{D}$ in $K^{+}$, then, as it 
was proved in [1], the set $\left\{\chi_{u(n)}: n \in \mathbb{N}_{0}\right\}$ of distinct characters on $\mathfrak{D}$ is a complete orthonormal system on $\mathfrak{D}$.

The Fourier transform $\widehat{f}$ of a function $f \in L^{1}(K) \cap$ $L^{2}(K)$ is defined by

$$
\widehat{f}(\xi)=\int_{K} f(x) \overline{\chi_{\xi}(x)} \mathrm{d} x .
$$

It is noted that

$$
\hat{f}(\xi)=\int_{K} f(x) \overline{\chi_{\xi}(x)} \mathrm{d} x=\int_{K} f(x) \chi(-\xi x) \mathrm{d} x .
$$

Furthermore, the properties of the Fourier transform on local field $K$ are much similar to those on the real line. In particular, Fourier transform is unitary on $L^{2}(K)$.

We now impose a natural order on the sequence $\{u(n)\}_{n=0}^{\infty}$. We have $\mathfrak{D} / \mathfrak{B} \cong \mathrm{GF}(q)$, where $\mathrm{GF}(q)$ is a $c$-dimensional vector space over the field $\operatorname{GF}(p)$. We choose a set $\left\{1=\zeta_{0}, \zeta_{1}, \zeta_{2}, \ldots, \zeta_{c-1}\right\} \subset \mathfrak{D}^{*}$ such that span $\left\{\zeta_{j}\right\}_{j=0}^{c-1} \cong \mathrm{GF}$ (q). For $n \in \mathbb{N}_{0}$ satisfying

$$
\begin{aligned}
0 \leq n<q, n=a_{0}+a_{1} p+\cdots+a_{c-1} p^{c-1}, \\
\quad 0 \leq a_{k}<p, k=0,1, \ldots, c-1,
\end{aligned}
$$

we define

$$
u(n)=\left(a_{0}+a_{1} \zeta_{1}+\cdots+a_{c-1} \zeta_{c-1}\right) \mathfrak{P}^{-1} .
$$

Also, for $n=b_{0}+b_{1} q+b_{2} q^{2}+\cdots+b_{s} q^{s}, n \in \mathbb{N}_{0}, 0 \leq$ $b_{k}<q, k=0,1,2, \ldots, s$, we set

$$
u(n)=u\left(b_{0}\right)+u\left(b_{1}\right) \mathfrak{P}^{-1}+\cdots+u\left(b_{s}\right) \mathfrak{P}^{-s} .
$$

This defines $u(n)$ for all $n \in \mathbb{N}_{0}$. In general, it is not true that $u(m+n)=u(m)+u(n)$. However, if $r, k \in \mathbb{N}_{0}$ and $0 \leq$ $s<q^{k}$, then $u\left(r q^{k}+s\right)=u(r) \mathfrak{P}^{-k}+u(s)$. Furthermore, it is also easy to verify that $u(n)=0$ if and only if $n=0$ and $\left\{u(\ell)+u(k): k \in \mathbb{N}_{0}\right\}=\left\{u(k): k \in \mathbb{N}_{0}\right\}$ for a fixed $\ell \in \mathbb{N}_{0}$. Hereafter, we use the notation $\chi_{n}=\chi_{u(n)}, n \geq 0$.

Let the local field $K$ be of characteristic $t>0$ and $\zeta_{0}, \zeta_{1}, \zeta_{2}, \ldots, \zeta_{c-1}$ be as above. We define a character $\chi$ on $K$ as follows:

$$
\chi\left(\zeta_{\mu} \mathfrak{P}^{-j}\right)= \begin{cases}\exp \left(\frac{2 \pi i}{j}\right), & \mu=0 \text { and } j=1, \\ 1, & \mu=1, \ldots, c-1 \text { or } j \neq 1 .\end{cases}
$$

In 2015, Shah [2] introduced the concept of frame multiresolution analysis (FMRA) on local fields, which can be sought as an extension of multiresolution analysis (MRA) on local fields of positive characteristic. First of all, let us recall the definition of FMRA as given by Shah. Let $K$ be a local field of positive characteristic $p>0$ and $\mathfrak{P}$ be a prime element of $K$. A frame multiresolution analysis (FMRA) of $L^{2}(K)$ is a sequence of closed subspaces $\left\{V_{j}: j \in \mathbb{Z}\right\}$ of $L^{2}(K)$ satisfying the following properties: (a) $V_{j} \subset V_{j+1}$, for all $j \in \mathbb{Z}$

(b) $\cup_{j \in \mathbb{Z}} V_{j}$ is dense in $L^{2}(K)$

(c) $\bigcap_{j \in \mathbb{Z}}^{j \in \mathbb{Z}} V_{j}=\{0\}$

(d) $f \in \mathbb{Z}(\cdot) \in V_{j}$ if and only if $f\left(\mathfrak{P}^{-1} \cdot\right) \in V_{j+1}, \quad$ for all $j \in \mathbb{Z}$

(e) There is a function $\varphi \in V_{0}$ such that $\{\varphi(\cdot-u(k)): k$ $\left.\in \mathbb{N}_{0}\right\}$ forms a frame in $V_{0}$

The function $\varphi$ is called a frame refinable function. It is noted that the shifts of $\varphi$ form a tight frame in the above FMRA. Replacing "a tight frame" in the above by "an orthonormal or a Riesz base" will arrive on the definition of a MRA on local fields of positive characteristic.

A finite family $\Psi:=\left\{\psi^{1}, \psi^{2}, \ldots, \psi^{L}\right\}$ generates a wavelet frame for $L^{2}(K)$ if there exist positive numbers $0<A \leq B<\infty$ such that, for all $f \in L^{2}(K)$,

$$
A\|f\|_{2}^{2} \leq \sum_{\ell=1}^{L} \sum_{j \in \mathbb{Z}} \sum_{k \in \mathbb{N}_{0}}\left|\left\langle f, \psi_{j, k}^{\ell}\right\rangle\right|^{2} \leq B\|f\|_{2}^{2}
$$

where $\psi_{j, k}^{\ell}=q^{j / 2} \psi^{\ell}\left(\mathfrak{P}^{-j} \cdot-u(k)\right)$. The largest constant $A$ and the smallest constant $B$ satisfying the above are called the lower and upper wavelet frame bound, respectively. A wavelet frame is a tight wavelet frame if $A$ and $B$ are chosen so that $A=B$, and then the set $\Psi:=\left\{\psi^{1}, \psi^{2}, \ldots, \psi^{L}\right\}$ is called a set of generators for the corresponding tight wavelet frame. Furthermore, the wavelet frame is called a Parseval wavelet frame if $A=B=1$, i.e.,

$$
\sum_{\ell=1}^{L} \sum_{j \in \mathbb{Z}} \sum_{k \in \mathbb{N}_{0}}\left|\left\langle f, \psi_{j, k}^{\ell}\right\rangle\right|^{2}=|f|_{2}^{2}, \quad \text { for all } f \in L^{2}(K),
$$

and in this case, every function $f \in L^{2}(K)$ can be written as

$$
f(x)=\sum_{\ell=1}^{L} \sum_{j \in \mathbb{Z}} \sum_{k \in \mathbb{N}_{0}}\left\langle f, \psi_{j, k}^{\ell}\right\rangle \psi_{j, k}^{\ell}(x) .
$$

A tight wavelet frame $\Psi$ is called a FMRA tight frame on local fields with frame bound 1 if $\Psi \subset V_{1}$. Here, in this article, we are concerned with a minimum-energy wavelet frame which is more restrictive than a FMRA tight frame on local fields of positive characteristic. Here, we recall the definition of minimum-energy wavelet frames on local fields of positive characteristic [3].

Definition 1. Let $\varphi \in L^{2}(K)$ satisfy $\widehat{\varphi} \in L^{\infty}, \widehat{\varphi}$ be continuous at 0 , and $\hat{\varphi}(0)=1$. Suppose that $\varphi$ generates the nested closed subspaces $\left\{V_{j}: j \in \mathbb{Z}\right\}$. Then, a finite family $\Psi=\left\{\psi^{1}, \psi^{2}, \ldots, \psi^{L}\right\} \subset V_{1}$ is called a minimum-energy wavelet frame associated with $\varphi$ if

$$
\sum_{k \in \mathbb{N}_{0}}\left|\left\langle f, \varphi_{1, k}\right\rangle\right|^{2}=\sum_{k \in \mathbb{N}_{0}}\left|\left\langle f, \varphi_{0, k}\right\rangle\right|^{2}+\sum_{\ell=1}^{L} \sum_{k \in \mathbb{N}_{0}}\left|\left\langle f, \psi_{0, k}^{\ell}\right\rangle\right|^{2}, \quad \text { for all } f \in L^{2}(K),
$$


where $\varphi_{j, k}(\cdot)=q^{j / 2} \varphi\left(\mathfrak{P}^{-1} \cdot-u(k)\right)$. By the Parseval identity, minimum-energy wavelet frame $\Psi$ must be a tight frame for
$L^{2}(K)$ with the frame bound being equal to 1 . At the same time, the above equation is equivalent to

$$
\sum_{k \in \mathbb{N}_{0}}\left\langle f, \varphi_{1, k}\right\rangle \varphi_{1, k}=\sum_{k \in \mathbb{N}_{0}}\left\langle f, \varphi_{0, k}\right\rangle \varphi_{0, k}+\sum_{\ell=1}^{L} \sum_{k \in \mathbb{N}_{0}}\left\langle f, \psi_{0, k}^{\ell}\right\rangle \psi_{0, k}^{\ell}, \quad \text { for all } f \in L^{2}(K) \text {. }
$$

Motivated and inspired by various constructions of minimum-energy wavelet frames [4-10] and classical wavelet frames on finite fields [11-13], we, in this paper, discuss some constructions of minimum-energy wavelet frames which are based on the frame multiresolution analysis on local fields of positive characteristic. This paper is organized in the following manner. In Section 2, we present some preliminaries for the FMRA and the minimum-energy wavelet frames on local fields of positive characteristic. In Section 3, we present the main results. Here, we provide a constructive algorithm for the existence of the minimumenergy wavelet frame on the local field of positive characteristic. We also construct an example to illustrate our algorithm.

\section{Notations and Preliminaries}

Here, we present some preliminaries for the FMRA and the minimum-energy wavelet frames on local fields of positive characteristic.

From the definition of FMRA on local fields, we know that $V_{0} \subset V_{1}=\overline{\operatorname{span}\left\{\varphi\left(\mathfrak{P}^{-1} \cdot-u(k)\right): k \in \mathbb{N}_{0}\right\}}$. Since $\varphi(\cdot) \in$ $V_{0}$, there exists a sequence $\left\{h_{k}\right\}_{k \in \mathbb{N}_{0}} \in l^{2}\left(\mathbb{N}_{0}\right)$ such that

$$
\varphi(x)=\sqrt{q} \sum_{k \in \mathbb{N}_{0}} h_{k} \varphi\left(\mathfrak{P}^{-1} x-u(k)\right) .
$$

The Fourier transform of (12) yields

$$
\widehat{\varphi}(\xi)=m_{0}(\mathfrak{P} \xi) \widehat{\varphi}(\mathfrak{P} \xi),
$$

where

$$
m_{0}(\xi)=\frac{1}{\sqrt{q}} \sum_{k \in \mathbb{N}_{0}} h_{k} \overline{\chi_{k}(\xi)}
$$

is an integral periodic function in $L^{2}(\mathfrak{D})$, where $\mathfrak{D}=$ $\{x \in K:|x| \leq 1\}$ is the ring of integers in $K$ and is often called the refinement symbol of $\varphi$. Observe that $\chi_{k}(0)=\widehat{\varphi}(0)=1$. Therefore, by letting $\xi=0$ in (13) and (14), we obtain $\sum_{k \in \mathbb{N}_{0}} h_{k}=1$.

Consider $\Psi=\left\{\psi^{1}, \psi^{2}, \ldots, \psi^{L}\right\} \subset V_{1}$, with

$\psi^{\ell}(x)=\sqrt{q} \sum_{k \in \mathbb{N}_{0}} h_{k}^{\ell} \varphi\left(\mathfrak{P}^{-1} x-u(k)\right), \quad \ell=1,2, \ldots, L$.

Equation (15) can be written in the frequency domain as

$$
\widehat{\psi}^{\ell}(\xi)=m_{\ell}(\mathfrak{P} \xi) \widehat{\varphi}(\mathfrak{P} \xi),
$$

where

$$
m_{\ell}(\xi)=\frac{1}{\sqrt{q}} \sum_{k \in \mathbb{N}_{0}} h_{k}^{\ell} \overline{\chi_{k}(\xi)}, \quad \ell=1,2, \ldots, L,
$$

are the integral periodic function in $L^{2}(\mathfrak{D})$ and are called the framelet symbols or wavelet masks.

With $m_{\ell}(\xi), \ell=0,1, \ldots, L$, as framelet symbols, we formulate the $q \times(L+1)$ matrix $\mathscr{M}(\xi)$ as

$$
\mathscr{M}(\xi)=\left(\begin{array}{cccc}
m_{0}(\xi+\mathfrak{P} u(0)) & m_{1}(\xi+\mathfrak{P} u(0)) & \cdots & m_{L}(\xi+\mathfrak{P} u(0)) \\
m_{0}(\xi+\mathfrak{P} u(1)) & m_{1}(\xi+\mathfrak{P} u(1)) & \cdots & m_{L}(\xi+\mathfrak{P} u(1)) \\
\vdots & \vdots & \ddots & \vdots \\
m_{0}(\xi+\mathfrak{P} u(q-1)) & m_{1}(\xi+\mathfrak{P} u(q-1)) & \cdots & m_{L}(\xi+\mathfrak{P} u(q-1))
\end{array}\right) .
$$

Shah and Debnath [3] proved that if $\Psi=\left\{\psi^{1}, \psi^{2}, \ldots\right.$, $\left.\psi^{L}\right\} \subset V_{1}$ forms a minimum-energy wavelet frame in $L^{2}(K)$, then the mask matrix $\mathscr{M}(\xi)$ should satisfy certain conditions as follows.

Lemma 1. Suppose that the refinable function $\varphi$ and the framelet symbols $m_{\ell}(\xi), \ell=0,1, \ldots, L$, satisfy (13)-(17). If $\hat{\varphi}$ is continuous at 0 and $\varphi(x)$ generates a sequence of nested closed subspaces $\left\{V_{j}: j \in \mathbb{Z}\right\}$, then the following statements are equivalent:

(1) $\Psi$ is a minimum-energy frame associated with $\varphi$.
(2) $\mathscr{M}(\xi) \mathscr{M}^{*}(\xi)=I_{q}, I_{q}$ is an identity matrix of order $q$ (19).

(3) $\begin{aligned} & \alpha_{r, s}=\sum_{k \in \mathbb{N}_{0}}\left\{h_{r-q k} h_{s-q k}+\sum_{\ell=1}^{L} h_{r-q k}^{\ell} h_{s-q k}^{\ell}\right\}-q \delta_{r, s}=0 \text {, } \\ & \forall r, s \in \mathbb{N}_{0} .\end{aligned}$

Lemma 1 gives the necessary and sufficient condition for the existence of the minimum-energy wavelet frames associated with refinable function $\varphi$. However, it is not a good choice to use this theorem to construct the minimum-energy wavelet frames. For convenience, Shah and Debnath [3] presented some conditions in terms of the framelet symbols. 
Lemma 2. Let $\varphi \in L^{2}(K)$ be the refinable function with refinement mask $m_{0}(\xi)$ such that $\hat{\varphi}$ is continuous at 0 and $\widehat{\varphi}(0)=1$. If $\Psi=\left\{\psi^{1}, \psi^{2}, \ldots, \psi^{L}\right\}$ is the minimum-energy wavelet frame associated with $\varphi$, then

$$
\sum_{r=0}^{q-1}\left|m_{0}(\mathfrak{P} \xi+\mathfrak{P} u(r))\right|^{2} \leq 1, \quad \text { for all } \xi \in K .
$$

In this paper, we start with the orthogonal vectors to obtain an explicit construction for the minimum-energy wavelet frame on local fields of positive characteristic.

\section{Main Results}

From Lemma 1, we know that if we want to obtain a minimum-energy wavelet frame on local fields of positive characteristic, we should find $L$ functions whose masks satisfy (19). Note the correlation of the rows of $\mathscr{M}(\xi)$; we should remove this feature first. For this, we introduce the polyphase decomposition technique. Similar to [3], we write

$$
\begin{aligned}
& m_{0}(\xi)=\frac{1}{\sqrt{q}}\left\{\overline{\chi_{u(0)}(\xi)} f_{1}(\xi)+\overline{\chi_{u(1)}(\xi)} f_{2}(\xi)+\cdots+\overline{\chi_{u(q-1)}(\xi)} f_{q}(\xi)\right\} \\
& m_{\ell}(\xi)=\frac{1}{\sqrt{q}}\left\{\overline{\chi_{u(0)}(\xi)} g^{\ell 1}(\xi)+\overline{\chi_{u(1)}(\xi)} g^{\ell 2}(\xi)+\cdots+\overline{\chi_{u(q-1)}(\xi)} g^{\ell q}(\xi)\right\}
\end{aligned}
$$

where $f_{r}(\xi)$ and $g^{\ell r}, r=1,2, \ldots, q$, are the polyphase decompositions of $m_{0}(\xi)$ and $m_{\ell}(\xi)$, respectively, and all these functions are $1 / q$-periodic. Let

$$
\begin{aligned}
\mathscr{P}(\xi) & =\left(\begin{array}{cccc}
f_{1}(\xi) & g^{11}(\xi) & \cdots & g^{\ell 1}(\xi) \\
f_{2}(\xi) & g^{12}(\xi) & \cdots & g^{\ell 2}(\xi) \\
\vdots & \vdots & \ddots & \vdots \\
f_{q-1}(\xi) & g^{1 q-1}(\xi) & \cdots & g^{\ell q-1}(\xi)
\end{array}\right), \\
\mathcal{S}(\xi) & =\left(\begin{array}{cccc}
\chi_{u(0)} & \chi_{u(1)} & \cdots & \chi_{u(q-1)} \\
\chi_{u(0)} & \chi_{\$ u(1)+u(1)} & \cdots & \chi_{\Re u(1)+u(q-1)} \\
\vdots & \vdots & \ddots & \vdots \\
\chi_{u(0)} & \chi_{\Re u(q-1)+u(1)} & \cdots & \chi_{\mathfrak{\$} u(q-1)+u(q-1)}
\end{array}\right) .
\end{aligned}
$$

Then, we can easily see that $\mathscr{M}(\xi)=(1 / \sqrt{q}) \mathcal{S}(\xi) \mathscr{P}(\xi)$, and (19) is equivalent to

$$
\mathscr{P}(\xi) \mathscr{P}(\xi)^{*}=I_{q} .
$$

The difference of (19) and (25) is that the rows of (25) are irrelevant to one another. Since $\left|m_{0}(\xi+\mathfrak{P} u(0))\right|^{2}+$ $\left|m_{0}(\xi+\mathfrak{P} u(1))\right|^{2}=\left|f_{1}(\xi+\mathfrak{P} u(0))\right|^{2}+\left|f_{2}(\xi+\mathfrak{P} u(0))\right|^{2}$, we have $\left|f_{1}(\xi+\mathfrak{P} u(0))\right|^{2}+\left|f_{2}(\xi+\mathfrak{\$} u(0))\right|^{2} \leq 1$.

Riesz lemma tells us that there can exist a function $f_{3}$ $(\xi+\mathfrak{P} u(0))$ such that $\left|f_{1}(\xi+\mathfrak{P} u(0))\right|^{2}+\left|f_{2}(\xi+\mathfrak{P} u(0))\right|^{2}$ $+\left|f_{3}(\xi+\mathfrak{\beta} u(0))\right|^{2}=1$. When $\left|f_{1}(\xi+\mathfrak{\beta} u(0))\right|^{2}+\mid f_{2}$ $\left.(\xi+\mathfrak{P} u(0))\right|^{2}=1$, then $f_{3}(\xi+\mathfrak{P} u(0))=0$, which is the special case of the orthonormal wavelet base on local fields of positive characteristic. With $f_{3}(\xi+\mathfrak{P} u(0))$ in hand, we can have a vector, in fact, a unit column vector, $\zeta_{0}=\left(f_{1}(\xi+\right.$ $\left.\mathfrak{P} u(0)), f_{2}(\xi+\mathfrak{P} u(0)), f_{3}(\xi+\mathfrak{P} u(0))\right)^{T}$. Now, we expect the existence of two more unit column vectors $\zeta_{1}$ and $\zeta_{2}$ such that

$$
\zeta_{0}^{T} \cdot \zeta_{1}=\zeta_{0}^{T} \cdot \zeta_{2}=\zeta_{1}^{T} \cdot \zeta_{2}=0 .
$$

In fact, $\zeta_{1}$ and $\zeta_{2}$ form an orthonormal fundamental system of solutions of the linear equation $\zeta_{0}^{T} \cdot x=0$. By straightforward calculation, an orthonormal fundamental system of solutions is

$$
\begin{aligned}
& \zeta_{1}=\left(-\frac{\overline{f_{3}}}{\Delta}, 0, \frac{\overline{f_{1}}}{\Delta}\right)^{T}, \\
& \zeta_{2}=\left(-\frac{f_{1} \overline{f_{2}}}{\Delta}, \Delta,-\frac{\overline{f_{2}} f_{3}}{\Delta}\right)^{T} .
\end{aligned}
$$

Here, $\Delta^{2}=\left|f_{1}\right|^{2}+\left|f_{3}\right|^{2}=1-\left|f_{2}\right|^{2}$. Here, we notice that if we choose $\mathscr{P}(\xi)$ as the first two rows of the following matrix

$$
\left(\zeta_{0}, \zeta_{1}, \zeta_{2}\right)=\left(\begin{array}{ccc}
f_{1} & -\frac{\overline{f_{3}}}{\Delta} & -\frac{f_{1} \overline{f_{2}}}{\Delta} \\
f_{2} & 0 & \Delta \\
f_{3} & \frac{\overline{f_{1}}}{\Delta} & -\frac{\overline{f_{2}} f_{3}}{\Delta}
\end{array}\right),
$$

then $\mathscr{P}(\xi)$ is a unitary matrix which implies that the corresponding matrix $\mathscr{M}(\xi)$ is a mask matrix. Moreover, the minimum-energy wavelet frame matrix $\mathscr{M}(\xi)$ on local fields has the shape

$$
\mathscr{P}(\xi)=\left(\begin{array}{ccc}
f_{1} & -\frac{\overline{f_{3}}}{\Delta} & -\frac{f_{1} \overline{f_{2}}}{\Delta} \\
f_{2} & 0 & \Delta
\end{array}\right) \mathcal{N}(\xi),
$$

where $\mathcal{N}(\xi)$ is a square matrix which is also unitary.

Given a refinement function $\varphi(x)$, the refinement mask $m_{0}(\xi+\mathfrak{P} u(0))$ should satisfy $m_{0}(\xi+\mathfrak{P} u(0))+m_{0}(\xi+$ $\mathfrak{P} u(1))+m_{0}(\xi+\mathfrak{P} u(2))=1$, which is the same as to the first column of (26). So, we can select the orthonormal wavelet masks as those of (26). So, all the orthonormal wavelet masks are of the shape 


$$
\left(\zeta_{0}, \zeta_{1}, \zeta_{2}\right)=\left(\begin{array}{ccc}
f_{1} & -\frac{\overline{f_{3}}}{\Delta} & -\frac{f_{1} \overline{f_{2}}}{\Delta} \\
f_{2} & 0 & \Delta \\
f_{3} & \frac{\overline{f_{1}}}{\Delta} & -\frac{\overline{f_{2}} f_{3}}{\Delta}
\end{array}\right)\left(\begin{array}{cc}
1 & 0 \\
0 & \mathcal{N}(\xi)
\end{array}\right) .
$$

\section{Example}

Let us consider the case of the Haar wavelet. The refinement function of the Haar wavelet on local fields will be given by $\varphi(x)=\chi_{\mathscr{D}}(x)$, and its refinement mask will be $m_{0}(\xi)=(1 / q)(1+\overline{\chi(\xi)})$. It can be observed that $m(\xi)$ satisfies $\left|m_{0}(\xi+\mathfrak{P} u(0))\right|^{2}+\left|m_{0}(\xi+\mathfrak{P} u(1))\right|^{2}=1$, and the corresponding polyphase decompositions are $f_{1}=f_{2}=1 / \sqrt{q}$. Here, we notice that the polyphase decompositions of the orthonormal wavelet masks $m_{\ell}(\xi)$ are $g^{\ell 1}(\xi)=-1 / \sqrt{q}$ and $g^{\ell 2}(\xi)=1 / \sqrt{q}$. Hence, the wavelet masks are given by $m_{\ell}(\xi)=-(1 / \sqrt{q})\left(1-\overline{\chi_{\ell}(\xi)}\right)$.

\section{Data Availability}

The data used to support the findings of this study are available upon request to the author.

\section{Conflicts of Interest}

The author declares that there are no conflicts of interest.

\section{Acknowledgments}

This work was supported by the UGC-BSR Research StartUp Grant (no. F.30-498/2019 (BSR)) provided by the UGC, Govt. of India.

\section{References}

[1] M. H. Taibleson, Fourier Analysis on Local Fields, Princeton University Press, Princeton, NJ, USA, 1975.

[2] F. A. Shah, "Frame multiresolution analysis on local fields of positive characteristic," Journal of Operators, vol. 2015, Article ID 216060, 8 pages, 2015.

[3] F. A. Shah and L. Debnath, "Minimum-energy wavelet frames on local fields," International Journal of Applied and Computational Mathematics, vol. 3, no. 4, pp. 3455-3469, 2017.

[4] Z. F. Li, "Construction of wavelet frames based on FMRA," Ph.D. thesis, China University, Yichang, China, 2013.

[5] J. J. Benedetto and S. Li, "The theorem of multiresolution analysis frames ansd application to filter banks," Applied and Computational Harmonic Analysis, vol. 5, pp. 398-427, Article ID HA970237, 1998.

[6] C. K. Chui and W. He, "Compactly supported tight frames associated with refinable functions," Applied and Computational Harmonic Analysis, vol. 8, no. 3, pp. 293-319, 2000.

[7] L. Debnath and F. A. Shah, Wavelet Transforms and Their Applications, Birkhäuser, Boston, MA, USA, 2015.

[8] X. Gao and C. Cao, "Minimum-energy wavelet frame on the interval," Science in China Series F: Information Sciences, vol. 51, no. 10, pp. 1547-1562, 2008.
[9] Y. Huang and Z. Cheng, "Minimum-energy frames associated with refinable function of arbitrary integer dilation factor," Chaos, Solitons \& Fractals, vol. 32, no. 2, pp. 503-515, 2007.

[10] F. Zhu, Q. Li, and Y. Huang, "Minimum-energy bivariate wavelet frame with arbitrary dilation matrix," Journal of Applied Mathematics, vol. 2013, Article ID 896050, 10 pages, 2013.

[11] A. G. Farashahi, "Generalized wavelet transforms over finite fields," Linear and Multilinear Algebra, vol. 68, no. 8, 2020.

[12] A. G. Farashahi, "Galois wavelet transforms over finite fields," Rocky Mountain Journal of Mathematics, vol. 49, no. 1, pp. 79-99, 2019.

[13] A. G. Farashahi, "Structure of finite wavelet frames over prime fields," Bulletin of the Iranian Mathematical Society, vol. 43, no. 1, pp. 109-120, 2017. 